\title{
Magnetism of CoPd self-organized alloy clusters on $\mathrm{Au}(111)$
}

P. Ohresser, E. Otero, F. Wilhelm, A. Rogalev, C. Goyhenex, L. Joly, H. Bulou, M. Romeo, V. Speisser, J. Arabski, G. Schull, and F. Scheurer

Citation: Journal of Applied Physics 114, 223912 (2013); doi: 10.1063/1.4846796

View online: https://doi.org/10.1063/1.4846796

View Table of Contents: http://aip.scitation.org/toc/jap/114/22

Published by the American Institute of Physics

\section{Articles you may be interested in}

A first-principles study on magnetocrystalline anisotropy at interfaces of Fe with non-magnetic metals Journal of Applied Physics 113, 233908 (2013); 10.1063/1.4811685

Large anisotropic Fe orbital moments in perpendicularly magnetized $\mathrm{C}_{2} \mathrm{FeAl}$ Heusler alloy thin films revealed by angular-dependent $\mathrm{x}$-ray magnetic circular dichroism

Applied Physics Letters 103, 102402 (2013); 10.1063/1.4819915

Spin engineering of CoPd alloy films via the inverse piezoelectric effect

Applied Physics Letters 82, 2458 (2003); 10.1063/1.1566795

Crossover between in-plane and perpendicular anisotropy in $\mathrm{Ta} / \mathrm{Co}_{x} \mathrm{Fe}_{100-x} / \mathrm{MgO}$ films as a function of $\mathrm{Co}$ composition

Journal of Applied Physics 113, 17C112 (2013); 10.1063/1.4799779

Exchange stiffness in thin film Co alloys

Journal of Applied Physics 111, 07 C919 (2012); 10.1063/1.3679433

$\mathrm{L}_{10}$ ordered phase formation in FePt, $\mathrm{FePd}$, CoPt, and CoPd alloy thin films epitaxially grown on $\mathrm{MgO}(001)$ single-crystal substrates

Journal of Applied Physics 111, 07 A708 (2012); 10.1063/1.3672856

\section{PHYSICS TODAY}

MANAGER'S GUIDE

WHITEPAPERS
Accelerate R\&D with

Multiphysics Simulation
READ NOW

PRESENTED BY

И $\subset$ M 


\title{
Magnetism of CoPd self-organized alloy clusters on $\mathrm{Au}(111)$
}

\author{
P. Ohresser, ${ }^{1}$ E. Otero, ${ }^{1}$ F. Wilhelm, ${ }^{2}$ A. Rogalev,${ }^{2}$ C. Goyhenex,${ }^{3}$ L. Joly, ${ }^{3}$ H. Bulou, ${ }^{3}$ \\ M. Romeo, ${ }^{3}$ V. Speisser, ${ }^{3}$ J. Arabski, ${ }^{3}$ G. Schull, ${ }^{3}$ and F. Scheurer ${ }^{3}$ \\ ${ }^{1}$ Synchrotron-SOLEIL, L'Orme des merisiers Saint-Aubin, BP 48, F-91192 Gif-sur-Yvette Cedex, France \\ ${ }^{2}$ European Synchrotron Radiation Facility (ESRF), BP 220, F-38043 Grenoble, France \\ ${ }^{3}$ Institut de Physique et Chimie des Matériaux de Strasbourg, UMR 7504, CNRS-Université de Strasbourg, \\ 23 rue du Loess, F-67034 Strasbourg, France
}

(Received 1 August 2013; accepted 27 November 2013; published online 13 December 2013)

\begin{abstract}
Magnetic properties of gold-encapsulated $\mathrm{Co}_{x} \mathrm{Pd}_{1-x}$ self-organized nano-clusters on $\mathrm{Au}(111)$ are analyzed by $\mathrm{x}$-ray magnetic circular dichroism for $x=0.5,0.7$, and 1.0. The clusters are superparamagnetic with a blocking temperature decreasing with increasing Pd concentration, due to a reduction of the out-of-plane anisotropy strength. No magnetic moment is detected on $\mathrm{Pd}$ in these clusters, within the detection limit, contrary to thick CoPd films. Both reduction of anisotropy and vanishing Pd moment are attributed to strain. (C) 2013 AIP Publishing LLC.
\end{abstract}

[http://dx.doi.org/10.1063/1.4846796]

\section{INTRODUCTION}

Combining alloy and dimensionality effects is expected to offer new engineering possibilities to optimize magnetic properties in nanoclusters, aiming at increased storage density. Presently, at the nanoscale, no stable magnetic information can be stored at room temperature because of too low superparamagnetic blocking temperatures, which scale with the product of magnetic volume and anisotropy. Therefore, magnetic alloy nanoparticles associating magnetic transition metals with high spin-orbit $4 d$ and $5 d$ elements are explored with the aim producing nanoparticles with enhanced anisotropy. Different routes of controlled-size nanocluster fabrication are explored today, mainly gas-phase cluster deposition ${ }^{1-3}$ and chemical wet synthesis. ${ }^{4-6}$ However, no efficient enhancement has been achieved so far. The most promising results were obtained recently on core-shell $\mathrm{FeCo}$ nano-islands grown on a $\mathrm{Pt}(111)$ surface, ${ }^{7}$ but the enhanced anisotropy was assigned to dimensionality effects rather than alloying. A way of benefiting from surface effects on welldefined size nanoparticles is to use self-organization on a patterned surface, as has been done for example for magnetic transition metals on reconstructed $\mathrm{Au}(111) .{ }^{8-10}$

Here we could produce bimetallic $\mathrm{Co}_{x} \mathrm{Pd}_{1-x}$ clusters on an $\mathrm{Au}(111)$, with a high quality of self-organization, similar to the one obtained for pure metals. We analyze the magnetic properties by x-ray magnetic circular dichroism (XMCD) for selected compositions of these bimetallic clusters. We first observe an enhanced orbital anisotropy in alloy clusters, but which does not correspond to an enhancement of the magnetocrystalline anisotropy, as one could have expected. The blocking temperature is actually reduced with increasing Pd concentration. We also observe a strong reduction of the Pd magnetic moment with respect to a bulk CoPd alloys, and which we attribute to strain, as supported by model density functional calculations.

\section{EXPERIMENTAL AND CALCULATION DETAILS}

Bimetallic clusters were prepared by co-deposition of $\mathrm{Co}$ and $\mathrm{Pd}$ on $\mathrm{Au}(111)$ single crystals held at $300 \mathrm{~K}$ in ultra-high vacuum. Electron-beam evaporators with integrated flux monitors were used to evaporate Co and Pd from high purity rods. The flux monitors were calibrated by depositing separately the pure elements in a sub-monolayer coverage on $\mathrm{Au}(111)$. The coverage was estimated by Scanning tunneling microscopy (STM). The evaporation rates of Co and Pd were adjusted to obtain the desired composition. Three samples were prepared, 0.25 monolayer (ML) of pure Co, 0.3 ML $\mathrm{Co}_{0.7} \mathrm{Pd}_{0.3}$, and $0.4 \mathrm{ML} \mathrm{Co}_{0.5} \mathrm{Pd}_{0.5}$. After characterization, they were covered by $2.5 \mathrm{~nm}$ gold, ensuring a continuous protective film of several monolayers, as verified by STM. As an XMCD reference for Pd $L_{2,3}$ edges, we fabricated in a separate installation by molecular beam epitaxy a $40 \mathrm{~nm}$ thick $\mathrm{Co}_{0.5} \mathrm{Pd}_{0.5}$ film deposited on a silicon substrate (following sequence was used: $\mathrm{Cr}(3 \mathrm{~nm}) / \mathrm{Co}(0.5 \mathrm{~nm}) / \mathrm{Co}_{0.5}$ $\left.\mathrm{Pd}_{0.5}(40 \mathrm{~nm}) / \mathrm{Co}(0.5 \mathrm{~nm}) / \mathrm{Cr}(20 \mathrm{~nm}) / \mathrm{Si}\right)$.

X-ray absorption and XMCD spectra at the $L_{2,3}$ Co edges were measured at the DEIMOS beamline at SOLEIL in total electron yield mode for light incidences from normal to grazing $\left(70^{\circ}\right)$ in a $6.5 \mathrm{~T}$ field applied along the photon beam. Magnetization loops at the maximum dichroism of the Co $L_{3}$ edge were recorded as a function of temperature, and averaged over the two light helicities (recording time: $\approx 40 \mathrm{~min}$ ). Absorption and XMCD spectra at $\mathrm{Pd} L_{2,3}$ edges were recorded at ID 12 at ESRF using an energy resolved fluorescence detector, with grazing light incidence onto the sample $\left(75^{\circ}\right)$, under an applied field of $6.0 \mathrm{~T}$ and at $7 \mathrm{~K}$.

$A b$ initio spin-polarized calculations were performed within the standard Kohn-Sham self-consistent density functional theory (DFT) using the SIESTA method ${ }^{11}$ in the generalized gradient approximation (GGA) with a Perdew Burke Emzerhof exchange-correlation potential. ${ }^{12}$ For the ion-electron interactions, the core electrons are replaced by norm-conserving pseudopotentials, ${ }^{13}$ while valence states are described using numerical atomic orbital basis sets (NAO). The electronic population is sorted for each orbital on each atomic site, using a Mulliken analysis.

To disentangle strain and hybridization effects, we have considered the following model configurations: the bulk 
phase $\mathrm{L} 1_{0}$ of a CoPd alloy, a chemically ordered CoPd(111) atomic layer inserted in a gold matrix in order to mimic the experimental $\mathrm{Au} / \mathrm{Co}_{0.5} \mathrm{Pd}_{0.5} / \mathrm{Au}(111)$ sample, and a $\mathrm{Co}(111)$ bilayer inserted in a gold matrix. For the Au-embedded layers, the simulation slabs are $6 \times 6$ atoms supercells with 3 to 6 layers thickness corresponding to 108 to 216 atoms. $\mathrm{Co}$ and CoPd embedded layers were considered to be accommodated to gold lattice parameter $(0.408 \mathrm{~nm})$ as optimized with DFT.

\section{RESULTS AND DISCUSSION}

Let us first summarize the most important features of the growth of bimetallic CoPd clusters, which is analyzed in detail elsewhere. ${ }^{14}$ Pure Co clusters are known to grow in a self-organized way as bilayers, ${ }^{15}$ whereas Pd grows as singlelayer clusters ${ }^{16,17}$ For Pd, although there is preferential nucleation on the kinks of the gold reconstruction, the organization of the cluster array is not as good as for Co. This is due to the fact that adsorption of $\mathrm{Pd}$ on $\mathrm{Au}(111)$ strongly disrupts the reconstruction, and hence nucleation sites. However, very few amounts $(\approx 3 \%)$ of cobalt co-deposited with Pd allow to stabilize the reconstruction and favor self-organization of Pd. It is therefore possible to obtain a high quality of self-organization of CoPd alloys for a very large composition range. ${ }^{14}$ Concerning the cluster height, Co forms bilayer whereas Pd forms single-layer high clusters, although the thermodynamically stable shape should lead to formation of bilayers for both elements. It was shown that for $\mathrm{Pd}$ on $\mathrm{Au}(111)$ atom hopping from first Pd to second layer is kinetically blocked by high energy activation barriers, whereas for Co, hopping is favored due to strain relaxation allowing clusters to reach the equilibrium shape. ${ }^{18}$ For alloy clusters, the height of the clusters depends on their composition but Pd has a strong tendency to stabilize the single-layer configuration. Up to about $x=0.5, \mathrm{Co}_{x} \mathrm{Pd}_{1-x}$ clusters are essentially single-layer high. Above $x=0.5$, bilayer clusters start to appear. At $x=0.7$, monolayer and bilayer clusters coexist ${ }^{14}$ (Fig. 1).

Figure 2 (left) shows the absorption spectra at the Co $L_{2,3}$ edges for the three samples, and the XMCD signal of the
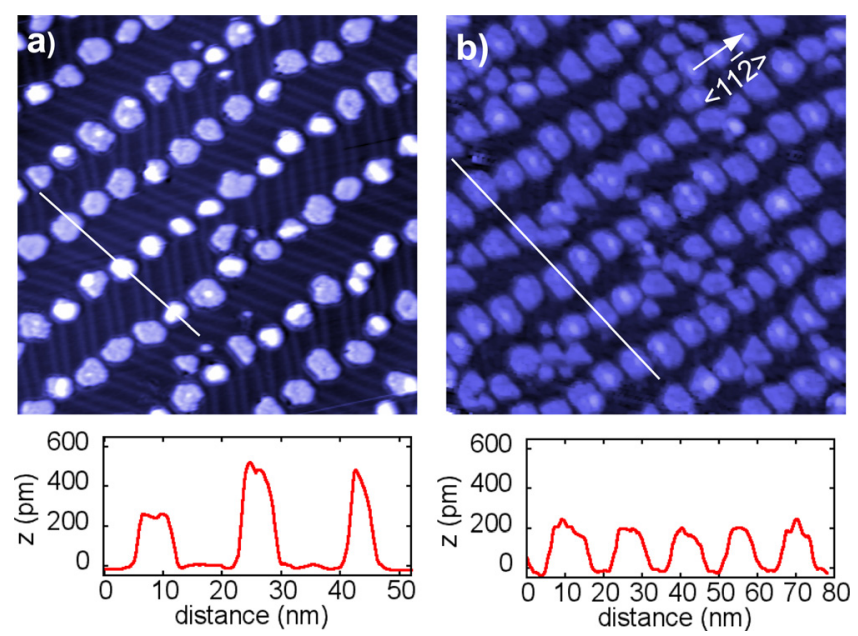

FIG. 1. (a) $0.30 \mathrm{ML} \mathrm{Co}_{0.7} \mathrm{Pd}_{0.3}(\mathrm{~V}=0.1 \mathrm{~V}, \mathrm{I}=50 \mathrm{pA})$; (b) $0.40 \mathrm{ML}$ $\mathrm{Co}_{0.5} \mathrm{Pd}_{0.5}(\mathrm{~V}=-0.6 \mathrm{~V}, \mathrm{I}=100 \mathrm{pA})$. Image size: $90 \mathrm{~nm}$. The line scans at the bottom correspond to white lines in the STM picture.

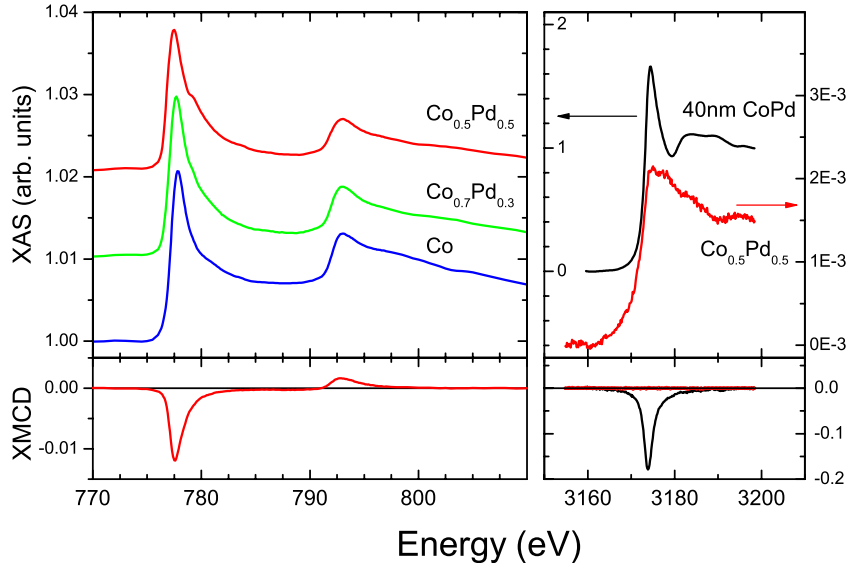

FIG. 2. Left: $\mathrm{Co} L_{2,3}$ absorption edges (white line, polarization averaged) for the pure Co (blue), $\mathrm{Co}_{0.7} \mathrm{Pd}_{0.3}$ (green), and $\mathrm{Co}_{0.5} \mathrm{Pd}_{0.5}$ (red) clusters. The XMCD signal is shown for $\mathrm{Co}_{0.5} \mathrm{Pd}_{0.5}$ clusters. Right: $\mathrm{Pd} \mathrm{L}$ absorption edge and corresponding $\mathrm{XMCD}$ for the $\mathrm{Co}_{0.5} \mathrm{Pd}_{0.5}$ clusters (red), and compared to the one of the $40 \mathrm{~nm}$ CoPd alloy film (black). The Pd XMCD spectra are shown on the same scale for comparison.

$\mathrm{Co}_{50} \mathrm{Pd}_{50}$ clusters. From the angular dependence of the $\mathrm{XMCD}$, the spin and orbital moments are deduced using the so-called sum rules. ${ }^{19,20}$ They are reported in Table I, together with the orbital moment anisotropy $\Delta m_{l}=m_{l}^{\perp}-m_{l}^{\|}$. The number of $d$ holes $\left(N_{d}\right)$ necessary to extract the absolute values of moments is determined by the DFT calculations (see Table II).

Looking at the right panel of Fig. 2, one notices that the $\mathrm{Pd} L_{3}$ edge is much broader and less intense for the $\mathrm{Co}_{0.5} \mathrm{Pd}_{0.5}$ clusters than for the $40 \mathrm{~nm}$ thick $\mathrm{Co}_{0.5} \mathrm{Pd}_{0.5}$ reference film. The Pd $L_{2}$ edge (not shown) is hardly detectable for the clusters: due to the very small quantity of Pd and to the overlap with the strong gold substrate background, we are at the limit of the detection possibility of the instrument. There is however no sizable XMCD signal at the $\operatorname{Pd} L_{3}$ edge in the clusters. Given the signal to noise ratio, we estimate by comparison with the reference film that the Pd magnetic moment in the clusters is below $\approx 0.1 \mu_{B}$. In the thick $\mathrm{Co}_{0.5} \mathrm{Pd}_{0.5}$ film, the $\mathrm{Pd}$ spin moment is $0.4 \mu_{B}$, comparable to the one obtained earlier in similar alloy films. ${ }^{21}$

Figure 3 shows XMCD magnetization loops at the Co $L_{3}$ edge for the three samples at grazing and normal light incidence for various temperatures. From the shape of the loops at $4 \mathrm{~K}$, one deduces that the easy magnetization axis is perpendicular to the surface for all samples. The loops were recorded along the easy axis as a function of temperature to estimate the blocking temperature, i.e., when there is a transition from a vanishing to a sizable coercive field. Loops

TABLE I. Blocking temperature, Co orbital moment perpendicular and parallel to the surface, orbital moment anisotropy, spin moment $\left(\mu_{B} /\right.$ atom). The number of $d$ holes obtained by DFT is $N_{d}=2.6$ (see Table II). The error on magnetic moments is in the order of $10 \%$.

\begin{tabular}{lcccrr}
\hline \hline Sample & $T_{B}$ & $m_{l}^{\perp}$ & $m_{l}^{\|}$ & $\Delta m_{l}$ & $m_{s}$ \\
\hline $0.25 \mathrm{ML} \mathrm{Co} / \mathrm{Au}(111)$ & $35 \mathrm{~K}$ & 0.22 & 0.21 & 0.01 & 2.1 \\
$0.30 \mathrm{ML} \mathrm{Co} 0.7 \mathrm{Pd}_{0.3} / \mathrm{Au}(111)$ & $25 \mathrm{~K}$ & 0.29 & 0.41 & -0.12 & 2.0 \\
$0.40 \mathrm{ML} \mathrm{Co} \mathrm{Co}_{0.5} \mathrm{Pd}_{0.5} / \mathrm{Au}(111)$ & $10 \mathrm{~K}$ & 0.44 & 0.34 & 0.10 & 1.7 \\
\hline \hline
\end{tabular}


TABLE II. DFT calculations of $\mathrm{Co}$ and Pd spin magnetic moments $\left(\mu_{B} /\right.$ atom $)$ for various model situations and number of $d$ holes.

\begin{tabular}{lccccc}
\hline \hline Model situation & Lattice constant $(\mathrm{nm})$ & $m_{s}^{C o}$ & $m_{s}^{P d}$ & $N_{d}^{C o}$ & $N_{d}^{P d}$ \\
\hline Bulk Co & 0.353 & 1.51 & $\ldots$ & 2.49 & $\ldots$ \\
Co (stretched) & 0.375 & 1.72 & $\ldots$ & 2.51 & $\ldots$ \\
Bulk Pd & 0.400 & $\ldots$ & 0.00 & $\ldots$ & 1.19 \\
Bulk CoPd & 0.382 & 1.96 & 0.25 & 2.59 & 1.10 \\
Bulk CoPd (stretched) & 0.410 & 2.20 & 0.19 & 2.63 & 1.08 \\
$\mathrm{Au} / 2 \mathrm{MLC} / \mathrm{Au}$ & 0.410 & 1.98 & $\ldots$ & 2.60 & $\ldots$ \\
$\mathrm{Au} / \mathrm{CoPd} / \mathrm{Au}$ & 0.410 & 2.32 & 0.09 & 2.62 & 1.08 \\
\hline \hline
\end{tabular}

recorded close to the blocking temperatures (reported in Table I), are also shown. One notices a clear reduction of the blocking temperature with increasing Pd concentration.

Considering the spin moments, for Co we observe in all cases a spin moment larger than in bulk Co $\left(1.59 \mu_{B}\right)$, and comparable to the one in bulk CoPd alloys $\left(1.95 \mu_{B}\right){ }^{22}$ For $\mathrm{Pd}$, we observe a vanishing moment, and this is rather unexpected, since Pd and Pt behave quite similarly and bear a large spin magnetic moments when alloyed with $\mathrm{Co}$ or $\mathrm{Fe}$, even in nanoparticles. ${ }^{4,21,23,24}$

Within the comparison of these results with DFT, some tendencies can be clearly identified. From Table II, we note that the magnetic moments are sensitive to both strain and chemical environment (see also Ref. 25): DFT predicts a larger moment for Co atoms either when stretched or in chemical vicinity of Pd. For Pd, the vicinity of Co in the alloy induces a sizable magnetic moment, but stretching of the alloy to epitaxial conditions on $\mathrm{Au}(111)$ reduces dramatically the Pd moment. Upon sandwiching the CoPd layer into gold, the Pd spin moment is even further reduced, in good agreement with the experiment. A slight discrepancy between experience and calculations remains: the Co moment is predicted to be larger for $\mathrm{CoPd}$ on $\mathrm{Au}(111)$ than for pure $\mathrm{Co}$ on $\mathrm{Au}(111)$. This may be due to the fact that the clusters are neither perfectly epitaxial, nor highly chemically ordered. Partial aggregation/demixing would also lead to a reduction of both Co and $\mathrm{Pd}$ spin moments with respect to

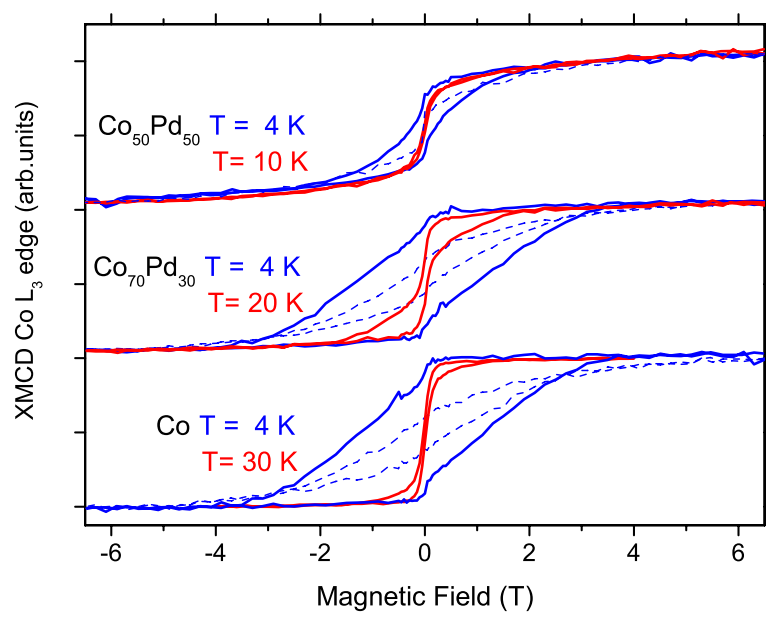

FIG. 3. XMCD magnetization loops at the Co $L_{3}$ edge at $4 \mathrm{~K}$ (blue) and a temperature close to the blocking temperature (red). Full lines: normal incidence; dashed lines: grazing incidence $\left(70^{\circ}\right)$. the model situations, as has been demonstrated in the case of FePt nanoclusters. ${ }^{5}$ The broadness of the $\mathrm{Pd}_{3}$ edge indeed suggests a distribution of composition. ${ }^{26}$

Turning to the orbital moment, we note that although magnetization loops all indicate out-of-plane anisotropy, the orbital moment anisotropy $\Delta m_{l}$ is either positive or negative. The simple correlation observed usually between the orbital moment anisotropy and the magnetocrystalline anisotropy $^{27,28}$ no more applies in strongly hybridized systems with large spin-orbit coupling, as emphasized by Andersson et al. ${ }^{29} \mathrm{In}$ such systems, as is the case for CoPd ${ }^{30}$ the magnetocrystalline anisotropy is no more a monotonous function of magnetic anisotropy. It is therefore not possible to deduce magnetocrystalline anisotropy tendencies by comparing the orbital moments of the different situations analyzed. We must therefore rely on the magnetization loops.

The decrease of the blocking temperature with Pd concentration, although unexpected, is in line with recent work which did neither evidence enhanced blocking temperatures in nano-alloy clusters combining transition metals with high spin-orbit elements. ${ }^{3,7}$ We obtain roughly the same order of magnitude for the anisotropy $(\approx 50 \mu \mathrm{eV} /$ atom $)$ as the one in CoPt nano-clusters, ${ }^{3}$ much less than in ordered bulk alloys. The decrease of the magnetic volume by replacing the magnetic Co atoms by weakly magnetic atoms like Pd or Pt is not compensated by a sufficient increase of anisotropy. In our particular case, a tentative explanation for the blocking temperature decrease for the mixed clusters with respect to pure Co clusters is the reduction of the out-of-plane anisotropy strength consecutive to a modified strain upon $\mathrm{Pd}$ incorporation. An increased in-plane parameter of Co leads to a reduced out-of plane anisotropy, as was demonstrated recently for Pt-Co and Au-Co core-shell clusters grown on $\mathrm{Au}(111))^{31,32}$ In case of pseudomorphic Co layers, the anisotropy was even predicted by calculations to be in-plane, ${ }^{33}$ and later verified for low-temperature grown Co films. ${ }^{34}$ In this latter case, the films are formed by small grains which may be able to accommodate high in-plane strain. Here, introducing Pd in the clusters tends to force Co in an environment with larger in-plane lattice constant, as shown by molecular dynamic calculations. ${ }^{14}$ Compared to pure Co clusters, which are almost relaxed, mixed clusters are much more strained. An indirect experimental evidence for this is the fact that the single-layer high clusters are favored with respect to pure Co clusters. Magneto-elastic effects hence favor in-plane magnetization, and the net result is a decrease of the out-of-plane anisotropy strength. Comparable effects were theoretically identified in CoPt nanostructures. ${ }^{35}$

\section{CONCLUSION}

In conclusion, we showed that in self-organized goldprotected $\mathrm{Co}_{x} \mathrm{Pd}_{1-x}$ nanoclusters on $\mathrm{Au}(111)$ the blocking temperature decreases with increasing Pd concentration. We attribute this effect to the decrease of the out-of-plane anisotropy strength consequently to high in-plane strain appearing in the clusters upon Pd introduction. The strain also strongly reduces the Pd spin moment with respect to bulk CoPd alloys, as supported by DFT calculations. Seeking enhanced 
anisotropy in nanoparticles will therefore require a fine tuning of strain and alloying effects to avoid antinomic behaviors.

\section{ACKNOWLEDGMENTS}

We are grateful to A. Barbier for providing an Au crystal, and to $\mathrm{H}$. Reichart for communicating recent results on NiPd. We thank B. Muller and J. G. Faullumel for valuable technical help. We acknowledge support from the Agence Nationale de la Recherche, program ANR-05-NANO-073 and computational time from the "Institut du Développement et des Ressources en Informatique Scientifique" (Project No. 090796).

${ }^{1}$ V. Dupuis, L. Favre, S. Stanescu, J. Tuaillon-Combes, E. Bernstein, and A. Perez, J. Phys.: Condens. Matter 16, S2231 (2004).

${ }^{2}$ A. Hillion, A. Cavallin, S. Vlaic, A. Tamion, F. Tournus, G. Khadra, J. Dreier, C. Piamonteze, F. Nolting, S. Rusponi, K. Sato, T. J. Konno, O. Proux, V. Dupuis, and H. Brune, Phys. Rev. Lett. 110, 087207 (2013).

${ }^{3}$ V. Dupuis, N. Blanc, L. E. Diaz-Sánchez, A. Hillion, A. Tamion, F. Tournus, and G. M. Pastor, Eur. Phys J. B 86, 83 (2013).

${ }^{4}$ C. Antoniak, J. Lindner, M. Spasova, D. Sudfeld, M. Acet, M. Farle, K. Fauth, U. Wiedwald, H.-G. Boyen, P. Ziemann, F. Wilhelm, A. Rogalev, and S. Sun, Phys. Rev. Lett. 97, 117201 (2006).

${ }^{5}$ C. Antoniak, M. Spasova, A. Trunova, K. Fauth, F. Wilhelm, A. Rogalev, J. Minár, H. Ebert, M. Farle, and H. Wende, J. Phys.: Condens. Matter 21, 336002 (2009).

${ }^{6}$ A. Smekhova, N. Atamena, D. Ciuculescu, P. Lecante, F. Wilhelm, C. Amiens, and A. Rogalev, J. Phys.: Conf. Ser. 200, 072091 (2010).

${ }^{7}$ S. Ouazi, S. Vlaic, S. Rusponi, G. Moulas, P. Buluschek, K. Halleux, S. Bornemann, S. Mankovsky, J. Minár, J. B. Staunton, H. Ebert, and H. Brune, Nat. Commun. 3, 1313 (2012).

${ }^{8}$ S. Padovani, I. Chado, F. Scheurer, and J. P. Bucher, Phys. Rev. B 59, 11887 (1999).

${ }^{9}$ P. Ohresser, N. B. Brookes, S. Padovani, F. Scheurer, and H. Bulou, Phys. Rev. B 64, 104429 (2001).

${ }^{10}$ N. Weiss, T. Cren, M. Epple, S. Rusponi, G. Baudot, S. Rohart, A. Tejeda, V. Repain, S. Rousset, P. Ohresser, F. Scheurer, P. Bencok, and H. Brune, Pys. Rev. Lett. 95, 157204 (2005).

${ }^{11}$ J. M. Soler, E. Artacho, J. D. Gale, A. García, J. Junquera, P. Ordejón, and D. Sánchez-Portal, J. Phys.: Condens. Matter 14, 2745 (2002).

${ }^{12}$ J. P. Perdew, K. Burke, and M. Ernzerhof, Phys. Rev. Lett. 77, 3865 (1996).
${ }^{13}$ N. Troullier and J. L. Martins, Phys. Rev. B 43, 1993 (1991).

${ }^{14} \mathrm{~F}$. Scheurer et al. (to be published).

${ }^{15}$ B. Voigtländer, G. Meyer, and N. M. Amer, Phys. Rev. B 44, 10354 (1991).

${ }^{16}$ A. W. Stephenson, C. J. Baddeley, M. S. Tikhov, and R. M. Lambert, Surf. Sci. 398, 172 (1998).

${ }^{17}$ C. S. Casari, S. Foglio, F. Siviero, A. Li Bassi, M. Passoni, and C. E. Bottani, Phys. Rev. B 79, 195402 (2009).

${ }^{18}$ H. Bulou, F. Scheurer, C. Goyhenex, V. Speisser, M. Romeo, B. Carrière, N. Moreau, V. Repain, C. Chacon, Y. Girard, J. Lagoute, S. Rousset, E. Otero, and P. Ohresser, Phys. Rev. B 87, 155404 (2013).

${ }^{19}$ T. Thole, P. Carra, F. Sette, and G. van der Laan, Phys. Rev. Lett. 68, 1943 (1992); P. Carra, B. T. Thole, M. Altarelli, and X. Wang, ibid. 70, 694 (1993).

${ }^{20}$ C. T. Chen, Y. U. Idzerda, H.-J. Lin, N. V. Smith, G. Meigs, E. Chaban, G. H. Ho, E. Pellegrin, and F. Sette, Phys. Rev. Lett. 75, 152 (1995).

${ }^{21}$ A. Vlachos, S. D. Pappas, V. Kapaklis, V. Karoutsos, A. Kordatos, F. Wilhelm, A. Rogalev, P. Fumagalli, P. Poulopoulos, M. J. Velgakis, and C. Politis, J. Nanosci. Nanotechnol. 12, 6240 (2012).

${ }^{22}$ H. P. J. Wijn (ed.), Data in Science and Technology (Springer Verlag, 1991).

${ }^{23}$ W. Grange, J.-P. Kappler, M. Maret, A. Rogalev, and J. Goulon, J. Synchrotron Radiat. 6, 679 (1999).

${ }^{24}$ P. Poulopoulos, M. Angelakeris, N. K. Flevaris, D. Niarchos, M. Nyvlt, V. Prosser, S. Visnovsky, Ch. Mueller, P. Fumagalli, F. Wilhelm, and A. Rogalev, J. Appl. Phys. 94, 7662 (2003).

${ }^{25}$ F. Aguilera-Granja, A. Vega, J. Rogan, X. Andrade, and G. García, Phys. Rev. B 74, 224405 (2006)

${ }^{26} \mathrm{H}$. Reichart, private communication (unpublished); In the case of $\mathrm{Ni}_{x} \mathrm{Pd}_{1-x}$ alloys, the $\mathrm{Pd} \mathrm{L}_{2,3}$ edges have been recently shown to be very sensitive to the chemical composition.

${ }^{27}$ P. Bruno, Phys. Rev. B 39, 865 (1989).

${ }^{28}$ G. van der Laan, J. Phys.: Condens. Matter 10, 3239 (1998).

${ }^{29}$ C. Andersson, B. Sanyal, O. Eriksson, L. Nordström, O. Karis, D. Arvanitis, T. Konishi, E. Holub-Krappe, and J. Hunter Dunn, Phys. Rev. Lett. 99, 177207 (2007).

${ }^{30}$ C. Goyhenex and G. Tréglia, Phys. Rev. B 83, 075101 (2011).

${ }^{31}$ Y. Nahas, V. Repain, C. Chacon, Y. Girard, J. Klein, J. Lagoute, G. Rodary, S. Rousset, H. Bulou, and C. Goyhenex, Phys. Rev. Lett. 103, 067202 (2009).

${ }^{32}$ P. Campiglio, N. Moreau, C. Chacon, J. Lagoute, Y. Girard, S. Rousset, H. Bulou, F. Scheurer, C. Goyhenex, P. Ohresser, E. Fonda, and H. Magnan, Phys. Rev. B 84, 235443 (2011).

${ }^{33}$ B. Z. Ujfalussy, L. Szunyogh, P. Bruno, and P. Weinberger, Phys. Rev. Lett. 77, 1805 (1996).

${ }^{34}$ S. Padovani, F. Scheurer, I. Chado, and J. P. Bucher, Phys Rev B 61, 72 (2000).

${ }^{35}$ G. Barcaro, L. Sementa, F. R. Negreiros, R. Ferrando, and A. Fortunelli, Nanoletter 11, 5542 (2011). 